\title{
Análisis económico y de la producción del descerdado manual de la fibra de llamas (Lama glama) Chaku
}

\author{
Quispe, E.C. ${ }^{1} ;$ Chipa $^{2}$ L. $^{2}$ y Pinares, R. ${ }^{2}$
}

'Departamento Académico de Medicina Veterinaria y Zootecnia. Universidad Nacional de Micaela Bastidas de Apurímac .UNAMBA. Abancay. Perú. 2Escuela Académico Profesional de Medicina Veterinaria y Zootecnia. UNAMBA. Abancay. Perú.

\section{PALABRAS CLAVE ADICIONALES}

Descerdado.

Fibra fina.

Camélido sudamericano.

\section{RESUMEN}

Con el objetivo de evaluar el efecto de las diferentes zonas del vellón de llama sobre el rendimiento al descerdado manual, así como para realizar una evaluación económica de dicho proceso, se esquilaron 15 llamas de tipo Chaku o lanudo 7 juveniles y 8 adultas, que corresponden a la primera esquila) de un Centro de Investigación ubicado en Huancavelica, Perú. Cada vellón fue extendido y dividido dos lados y en 7 zonas corporales. Posteriormente en el laboratorio, previo pesado de cada zona, las muestras fueron descerdadas manualmente obteniéndose tres componentes: Fibra fina, fibra gruesa e impureza, los cuales también fueron pesadas. Asimismo 7 personas previamente entrenadas, realizaron el descerdado de muestras de 7 zonas corporales de 10 vellones previo control del tiempo, a fin de obtener el rendimiento de fibra fina/h. Los datos fueron procesados mediante un diseño completamente al azar y un diseño en bloque completamente al azar con arreglo factorial de $7 x 2$ y para comparar medias se utilizaron pruebas de Tukey y Duncan. Los resultados muestran que al descerdado manual de la fibra de llama se obtiene un alto rendimiento de fibra fina $(68,60 \pm 2.54 \%)$, y que el estrato etáreo no tiene efecto sobre el rendimiento porcentual, pero si sobre el rendimiento de producción, mientras que las zonas de donde se puede obtener mayor cantidad de fibra fina son las que corresponden a las tres zonas del manto (anterior, central y posterior) y al cuello. También se ha encontrado que no existe efecto de la zona corporal sobre al rendimiento/persona/h y que dicha eficiencia por los descerdadores no es buena $(9,9 \pm 1,1 \mathrm{~g} /$ persona/h), por lo que al análisis económico resulta que la producción de $1 \mathrm{~kg}$ de fibra fina al descerdado manual tendría un costo en mano de obra de 141,45 USD \$, y que considerando los costos variables, $1 \mathrm{~kg}$ de fibra fina de llama costaría 145,92 USD \$, lo que contrasta con el precio de fibra fina de llama que se paga en el mercado, lo cual apenas llega a los 7,14 USD \$, lo que permite concluir que el descerdado manual de fibra de llama tiene un alto costo económico.

\section{Analysis of the productivity and economics of manual deharing of Chaku llama (Lama glama)}

\section{SUMMARY}

The aim of this preliminary investigation was to quantify the components of Chaku llama fleeces, the time taken to separate the fine fiber component and to estimate the economics of the manual dehairing process. Fleece samples were obtained from 8 adult and 7 young llama grazing at Huancavelica, Peru. Each fleece was divided into the two sides and then into 7 areas corresponding to the body sites. Later in the laboratory the samples were manually sorted into three components: fine fiber, coarser fibers and impurities, which were weighed. The time taken for 7 previously trained people to performed the manual fiber separation of all samples from 10 fleeces was recorded. The data analysed using 7 sampling site $\times 2$ age factorial design. Tukey and Duncan tests were used to compare means. The mean fine fiber yield was higher for the saddle (anterior, central and posterior) and neck sites (69-73\%) compared with the remainder of the fleece $(64-65 \%)$. Age of llama did not affect fine fiber yield. However younger llama produced lighter fleeces compared with adult llama. While there was no effect of fleece site on the average rate of fiber dehairing $19.9 \mathrm{~g} / \mathrm{h}, 79 \mathrm{~g} /$ day), there was a large range between people and samples (4-19 $\mathrm{g} / \mathrm{hour})$. This suggests that there is a combination of samples and people where the rate of fiber separation is twice the average. Economic analysis showed tha labor cost for manual separation of $1 \mathrm{~kg}$ of fine fiber was 141.45 USD \$, which contrasts with the market price of fine llama of 7.14 USD \$. Including all costs, the ratio of benefits/costs was 0.05 . It was concluded that the manual dehairing of llama fiber was not economic, and for economic development it is necessary to develop mechanical dehairing processes. 


\section{INTRODUCCIÓN}

La llama (Lama glama) puede alcanzar un peso adulto de 80 a $150 \mathrm{~kg}$, y por ende es el camélido sudamericano de mayor tamaño (Bustamante et al., 2006), su utilización es dirigida para el transporte y el abastecimiento de carne, pero también produce fibra que tiene diversos usos. Existen dos razas, Chaku, Lanuda o Tampulli y Ccara o Pelada (Valbonesi et al., 2010), diferenciándose una de otra por la magnitud de cobertura del cuerpo, pues mientras que Chaku tiene mayor cobertura de fibra, incluyendo las extremidades, Ccara tiene una apariencia de mayor fortaleza corporal con poca cobertura de fibras en el cuerpo y extremidades. Existen también tipos intermedios que pueden confundirse con el Huarizo, ésta última producto del cruce de llama con alpaca, que ocurre frecuentemente en sistemas de crianza mixta como es el caso de la mayoría de pequeños productores (Rodríguez, 2007, Valbonesi et al., 2010).

A pesar que Perú es el segundo productor mundial de llamas con alrededor de 1,5 millones de animales y una producción de 760 ton/año (Bolivia alberga cerca de 2,5 millones de llamas que producen 433 ton), la fibra producida por ellos es poco aprovechada (Quispe et al., 2009), debido a que muchos criadores no esquilan sus llamas, y la poca cantidad de fibra que se esquila es utilizada para el consumo doméstico y un pequeño porcentaje es comercializado en mercados locales. Los compradores y productores de fibra coinciden en que existe una demanda de fibra de llama, pero por razones de bajos índices de extracción, fluctuación de calidad y cantidad, no se aprovecha este potencial (Stemmer et al., 2005). En la actualidad, en la zona central del Perú, es común ver animales con vellones de dos, tres o más años, debido que los productores no esquilan sus animales anualmente bajo el argumento equivocado que el largo de la fibra es insuficiente de acuerdo a los requerimientos de la industria textil, pues la longitud de fibra alcanzada al año varían entre 6,2 a $8,5 \mathrm{~cm}$ (Cardozo, 2007; Siguayro, 2009) a pesar que a la fecha ya existe tecnología para el procesamiento de fibras de longitudes hasta de $2 \mathrm{~cm}$, bajo el sistema de cardado (Nibikora y Wang, 2010). Otra justificación de la falta de esquila se debería a la falta de una comercialización organizada (Quispe et al., 2009).

Corrientemente se ha indicado que las llamas producen vellones con fibras heterogéneas de baja calidad, sin embargo el vellón está compuesto principalmente por dos capas: una capa externa conformada por fibras gruesas (denominada comúnmente cerdas) que le protegen de los rayos solares, la lluvia y el polvo, y una capa interna con fibras finas (down) que forma una capa aislante (Dalton y Franck, 2000), siendo ésta última de buena calidad, y que su uso estaría acondicionado al proceso de descerdado (McGregor, 2012).

Los caracteres que determinan la calidad de la lana y de la fibra están en función a los requerimientos de la industria textil, en los que influyen el diámetro , color, longitud de la fibra, la variabilidad entre fibras (evaluado a través del coeficiente de variación), perfil del diámetro de fibra (variación del diámetro a lo largo de la fibra), resistencia a la tracción, factor de confort, entre otros (Lupton et al., 2006), los cuales mejoran ostensiblemente mediante el descerdado (proceso consistente en la extracción o eliminación de la fibra gruesa o pelos del conjunto de las fibras que componen el vellón), ya que se reduce el diámetro medio de la fibra, su coeficiente de variación y la finura al hilado, incrementándose a la vez el factor de confort, e índice de curvatura, tal como lo determinaron en cashemere McGregor y Butler (2008) y en llamas Pinares et al. (2014), así como también reduce la sensación de picazón fundamentalmente en vellones de doble capa, siendo menos dicha reducción en vellones de capa simple (Frank et al., 2011).

Las fibras gruesas, producto del descerdado, también puede utilizarse de diferentes maneras, así Frank (2011) considera que debe otorgarse un buen valor a las fibras gruesas, por lo que se debiera dejar de utilizar el término subproducto, pues éstas fibras mezcladas con lanas de $>30 \mu \mathrm{m}$, dan lugar alfombras con una buena resiliencia y resistencia a la abrasión, mientras que las fibras más finas, se prestan a la fabricación, pura o en mezcla con lana, de tejidos para saco sport, sobretodo, tapados y decoración de alta calidad. El peso de vellón sucio es de 1185,9 g con una desviación estándar de 406,7 g (Cochi, 1999). El rendimiento de fibra fina al descerdado se encuentra influenciado por el tipo de descerdado, tipo de maquinaria, la especie animal (Hopkins, 1993), lugar de crianza (Cochi, 1999), el tipo de vellón (Frank et al., 2012), finura, entre otros. Así Cardozo et al. (2007), proporcionan información en base a 124 llamas estudiadas, y encontraron que el vellón fino está en una proporción de $67,88 \%$, bragas en un $30,04 \%$ y los pedazos (khopa) en un 2,08\%. De otro lado Frank et al. (2012) indican que los vellones de llama de doble capa tienen un rendimiento en top luego del descerdado alrededor de 40,59\%, que resulta ser bajo en comparación con vellones de tipo intermedio, de una sola capa, vellón semi lustroso y vellón lustroso, los cuales arrojan rendimientos de 63,21\%, 54,66\%, 58,91\% y $60,83 \%$ respectivamente, mientras que Stemmer et al. (2005), en llamas de Ayopaya (Bolivia) encontraron una proporción de fibras finas de $91,3 \%$ con un diámetro de 20,47 $\mu \mathrm{m}$. Con respecto a vellones de otras especies, Adot et al. (2008), reportaron que vellones de vicuñas que son descerdadas manualmente, dan rendimientos alrededor del $70 \%$ y a la vez, Sacchero y Mueller (2005) encontraron rendimientos alrededor del $77 \%$; mientras que McGregor (2004) al considerar lotes comerciales de cashemere, indica un rendimiento de fibra fina promedio de $33,3 \%$.

El descerdado, puede ser manual (utilizando pinzas o dedos para la extracción de fibras gruesas), o mecánico (basado en el uso de la fuerza centrífuga y por flotación en corriente de aire). Este proceso se realiza en la fibra de especies como la vicuña, guanaco, llama, alpaca, cashemere, conejo de Angora, liebre, yak, búfalo, buey almizclero, camello, y entre otros (Hopkins, 1993). El descerdado mecánico es justificado cuando existen grandes cantidades de materia prima para procesar, resultando práctico y económico para el caso del cashemere (Adot et al., 2008) que tiene una producción de más de 8 mil ton, sin embargo cuando la producción es baja, como el caso de fibra de vicuña 
y guanaco, generalmente el descerdado se realiza en forma manual (McGregor, 2012), generándose nuevas fuentes de empleo y nuevos flujos de ingresos económicos (Siguayro, 2009).

Así, para el caso de la fibra de llama, el descerdado manual beneficiaría principalmente a las familias de bajos recursos económicos y a los mismos productores (Rodríguez, 2007), aunque en la actualidad existe aún poco conocimiento respecto a la producción de fibra fina al descerdado, resaltando el trabajo de Cochi (1999), quien reportó un rendimiento entre 1.3 a 2.1 $\mathrm{kg}$ /persona/día que deriva un costo de 3,63 USD\$). Sin embargo debido que existe diferencias entre las diferentes zonas del vellón tal como fue demostrado en llamas por Martínez et al. (1997), en alpacas por McGregor et al. (2012) y en vicuñas por Quispe et al. (2014), resultaría lógico suponer que la zona del vellón tendría efecto sobre el rendimiento al descerdado de la fibra de llama, sin embargo esto no ha sido aún demostrado. Por dichas razones se ha realizado el presente trabajo con los objetivos de conocer el rendimiento al descerdado (en función a porcentaje, cantidad y producción por unidad de tiempo), evaluar el efecto de la edad y las zonas corporales sobre estas variables y realizar una evaluación económica de dicho proceso.

\section{MATERIAL Y MÉTODOS}

\section{LOCALIZACIÓN Y MUESTRAS UTILIZADAS}

Se utilizaron 15 vellones de llamas Chaku (adultas, mayor a 2 años= 7; juveniles, de 1 a 2 años $=8$ ) criadas en el Centro de Investigación de Camélidos Sudamericanos (CICS) - Lachocc de la Universidad Nacional de Huancavelica (UNH), ubicado a una altitud de 4800 $\mathrm{msnm}$, con temperaturas que varían desde $-5^{\circ} \mathrm{C}$ hasta $18^{\circ} \mathrm{C}$ y con una precipitación pluvial que alcanza los 752,4 mm/año. La esquila se realizó entre los meses de octubre y diciembre del 2012, utilizándose tijeras de esquila (lapiacos), para lo cual se colocaron a los animales en posición lateral y sujetando a los animales de las dos extremidades anteriores y las posteriores y mediante trabas (sogas de media pulgada de grosor traspasadas en la parte terminal de un trozo de madera de 12 pulgadas de largo), de modo que una vez obtenido el producto se realizó el envellonado (con presentación tipo tambor), ensacado y rotulado.

\section{ProcedimIENTO DEL DESCERDADO Y OBTENCIÓN DE DATOS}

Posteriormente, los vellones fueron trasladados al Laboratorio de Lanas y Fibras de la Facultad de Medicina Veterinaria y Zootecnia de la Universidad Nacional Micaela Bastidas de Apurímac, localizada en la ciudad de Abancay, ubicado en la parte Sur Centro del Perú, , que se encuentra a 2378 msnm; donde cada vellón luego de someterse al desenvellonado en una mesa de madera de $2 \times 3 \mathrm{~m}$, fue dividido en dos lados (derecho e izquierdo), y luego cada lado fue seccionado en siete zonas: manto anterior (MaA), manto central $(\mathrm{MaC})$, manto posterior $(\mathrm{MaP})$, cuello, miembro anterior (MiA), miembro posterior (MiP) y barriga, de acuerdo a la metodología utilizada por McGregor et al. (2012), para luego ser pesadas en una balanza analítica y colocadas en bolsas de polietileno, obteniéndose así 14 submuestras de cada vellón.

Cada una de las 210 submuestras fueron sometidas al proceso del descerdado manual, de acuerdo al procedimiento descrito por Frank et al. (2012), el cual que está basado en la utilización de los dedos en lugar de fórceps, con 5 a 8 movimientos de descerdado por mechón. Para una mejor separación de las fibras gruesas, se utilizó dos fondos de papel como contraste: el fondo blanco ayudó a ubicar fibras gruesas de color negro y café oscuro; y el fondo negro para fibras gruesas blancas y café claro. De cada submuestra descerdada se obtuvieron tres componentes: Fibra fina (llamada también down, que corresponde a lo que queda después de extraer las fibras gruesas, y que tienen buena finura), fibra gruesa (llamada también pelos o cerdas) e impureza (compuesta por fibras muy cortas, restos vegetales y tierra), los cuales fueron pesados en una balanza analítica, cuya información fue registrada, considerándose la identificación del animal, estrato etáreo y región corporal. A fin de evaluar la efectividad del descerdado se evaluó 5 características textiles (media de diámetro de fibra - MDF, coeficiente de variación de la MDF - CVMDF, factor de confort - FC, índice de curvatura - IC y finura al hilado - FIHI) antes y después del descerdado a fin de calcular la variación al descerdado mediante la diferencia entre los resultados antes y después del descerdado para cada una de las características.

Para determinar el rendimiento al descerdado expresado en $\mathrm{g} /$ persona/h, se consideraron 7 personas, a quienes se les entrenaron durante 5 días previos al experimento, luego se procedió al descerdado de las fibras, utilizando el procedimiento descrito por Frank et al. (2012). A cada persona se entregó una muestra de 100 g previa aleatorización de las zonas corporales y luego de transcurrida dos horas (desde las 08:00 a las 10:00 horas) se pesó la fibra fina obtenida por cada person, ponderándose luego los resultados por hora. Cada día se trabajó solamente con 1 vellón. El análisis económico del descerdado pasó por la obtención de la cantidad de fibra por mes (teniendo en cuenta el rendimiento/persona/h y rendimiento/persona/día de 8 horas de trabajo efectivo) considerando como remuneración correspondiente al salario mínimo vital en Perú, expresado en USD \$, para luego calcularse las utilidades y la relación de Beneficio/Costo.

\section{ANÁLISIS ESTADÍSTICO}

Los datos obtenidos se procesaron mediante el paquete estadístico $\mathrm{R}$ versión 3.1.1. (R Core Team, 2014) iniciándose con un análisis exploratorio univariado, pasando luego por las prueba de Shapiro y de Barlett para evaluar la normalidad de los residuos estandarizados y la homogeneidad de varianzas de los datos. Ningún dato fue descartado, pero para los cálculos de los diferentes estadísticos y uso de los diversos diseños experimentales, previamente los datos expresados en porcentajes fueron sometidos a una transformación trigonométrica.

A fin de evaluar el rendimiento al descerdado en unidades absolutas y relativas se utilizó estadística descriptiva, calculándose los principales estadísticos 
Tabla I. Promedio \pm error estándar (ee) de la fibra antes del descerdado y estadísticos de las variaciones al descerdado de cinco características textiles de vellones de llama Chaku (Average \pm standard error (se) of fiber before dehairing and statistics of variation after dehairing of five textile characteristics at Chaku llama fleeces).

\begin{tabular}{|c|c|c|c|c|c|}
\hline \multirow{2}{*}{ Características textiles } & \multirow{2}{*}{ Promedio antes del descerdado } & \multicolumn{4}{|c|}{ Estadísticos de variación al descerdado } \\
\hline & & Promedio & Mínimo & Máximo & Error estándar \\
\hline Media de diámetro de fibra $(\mu \mathrm{m})$ & $26,42 \pm 0,42$ & $-4,21$ & $-0,34$ & $-9,79$ & 0,19 \\
\hline Coeficiente de variación (\%) & $22,50 \pm 0,34$ & $-2,51$ & $-0,01$ & $-8,11$ & 0,17 \\
\hline Factor de confort (\%) & $76,84 \pm 1,37$ & 16,13 & 1,43 & 38,01 & 0,69 \\
\hline Indice de curvatura ( $\%$ mm) & $30,73 \pm 1,71$ & 4,75 & 0,30 & 11,38 & 0,22 \\
\hline Finura al hilado $(\mu \mathrm{m})$ & $26,48 \pm 0,45$ & $-5,02$ & $-0,37$ & $-11,17$ & 0,23 \\
\hline
\end{tabular}

mediante el paquete $p s y c h$ de R. La evaluación del efecto del estrato etáreo sobre el rendimiento al descerdado expresado en valores absolutos y relativos de peso de la fibra de llama pasó por un Diseño Completamente al Azar (DCA), y la prueba de Tukey sirvió para la comparación de medias. Posteriormente se elaboraron los gráficos respectivos, mediante el paquete multcom de R. Para evaluar el efecto de la zona corporal sobre el rendimiento al descerdado se utilizó el Diseño en Bloque Completamente al Azar con Arreglo factorial de $7 \times 2$, considerando como bloque a los animales, y como factores la región (en 7 niveles) y el lado (en dos niveles: Izquierda y Derecha). Finalmente para la determinación del efecto de la región corporal sobre la cantidad obtenida de fibra fina obtenida/persona/h se utilizó un DCA, y la prueba de Duncan permitió realizar la comparación de medias entre las diferentes zonas del vellón.

\section{RESULTADOS}

\section{RENDIMIENTO POR VELLÓN DE FIBRA FINA, CERDA E IMPUREZA}

Inicialmente se obtuvo $1,71 \pm 0,27 \mathrm{~kg}$ (promedio \pm error estándar) de fibra/vellón de llama Chaku, con una finura de $26,42 \pm 0,42 \mu \mathrm{m}$, que luego del descerdado manual disminuyó en 4,21 $\pm 0,19 \mu \mathrm{m}$, encontrándose también variaciones de otras características textiles (CVMDF, FC, IC, y FIHI), que comprueban la efectividad del descerdado manual en la mejora de la calidad de la fibra (tabla I).

Luego del proceso de descerdado (tabla II), la fibra fina fue el componente más abundante del vellón, alcanzando más de los dos tercios, correspondiendo

Tabla II. Rendimiento al descerdado manual de fibra fina, cerda e impurezas en vellones de llama Chaku (The yield to the manual dehairing of down fiber, coarser fiber and impurities on Chaku llama fleeces).

\begin{tabular}{lcccccr}
\hline Componentes & N & Promedio & DE & $m$ & M & \multicolumn{1}{c}{ EE } \\
\hline Fibra fina (\%) & 15 & 68,60 & 9,84 & 51,74 & 83,61 & 2,54 \\
Cerda (\%) & 15 & 24,67 & 8,96 & 10,18 & 41,10 & 2,31 \\
Impurezas (\%) & 15 & 6,73 & 3,99 & 1,55 & 16,28 & 1,03 \\
Fibra fina (g) & 15 & 1184,5 & 786,6 & 412,4 & 3345,3 & 203,1 \\
Cerda (g) & 15 & 404,9 & 273,7 & 140,1 & 1155,6 & 70,7 \\
Impurezas (g) & 15 & 124,3 & 118,5 & 24,8 & 409,4 & 30,6 \\
Total (g) & 15 & 1713,7 & 1028,6 & 706,3 & 4427,3 & 265,6 \\
\hline
\end{tabular}

$\mathrm{DE}=$ Desviación estandar; $\mathrm{m}=$ Mínimo; $\mathrm{M}=$ Máximo; $\mathrm{EE}=\mathrm{Error}$ estandar. a la fibra gruesa sólo la cuarta parte, mientras que las impurezas representan una mínima proporción; consecuentemente la cantidad de producto aprovechable por la industria textil y/o artesanal (fibras finas y gruesas) se encuentra alrededor del $93 \%$. Debe precisarse que estas proporciones y la cantidad total de fibra corresponden a llamas que han sido esquiladas por primera vez, habiéndose encontrado inclusive llamas adultas hasta con 6 años de crecimiento de fibra con un alto peso de vellón $(4,43 \mathrm{~kg})$, aunque también se encontraron animales de un año con vellones de $0,71 \mathrm{~kg}$, lo que representa un amplio rango (cuantificado en más del $60 \%$ de coeficiente de variación). Contrariamente a ello, cuando se evalúa el rendimiento de fibra fina en función a porcentaje la variación no es alta según el estrato etáreo, pues alcanza un coeficiente de variación menor al $4 \%$, lo que nos conlleva a indicar que los vellones de llamas Chaku, aún cuando tengan diferentes pesos, tienen una proporción homogénea de fibra fina $(68,60 \pm 2,54 \%)$.

\section{EFECTO DEL ESTRATO ETÁREO Y DE LA ZONA CORPORAL SOBRE EL RENDIMIENTO EN FIBRA FINA, CERDA E IMPUREZA}

El porcentaje de fibra fina, fibra gruesa e impureza obtenidos al descerdados no resulta influido por el estrato etáreo (figura 1: izquierda), alcanzándose rendimientos de: fibra fina de $68,91 \pm 0,01$ contra $68,32 \pm 0,01$; fibra gruesa $23,19 \pm 0,01$ contra $25,96 \pm 0,01$; e impureza de 7,89 contra $5,71 \pm 0,01 \%$ para llamas adultas y juveniles, respectivamente. Sin embargo cuando se evalúa el rendimiento de dichos componentes en unidad de peso $(\mathrm{g})$, se ha encontrado que los animales adultos producen mayor cantidad de fibra que los animales jóvenes (figura 1: derecha). De este modo la cantidad de fibra fina, cerda e impurezas producida por llamas adultas es de $1,77 \pm 0,30 ; 0,57 \pm 0,12$ y $0,20 \pm 0,05 \mathrm{~kg}$, mientras que las llamas jóvenes producen $0,78 \pm 0,08$; $0,26 \pm 0,04$ y $0,06 \pm 0,02 \mathrm{~kg}$ respectivamente para cada componente.

Asimismo, se ha encontrado suficiente evidencia para afirmar que la región corporal tiene efecto sobre el porcentaje y cantidad de fibra fina y gruesa obtenida al descerdado manual, hallándose que la zona del vellón que tiene mayor proporción de fibra fina y menor proporción de fibra gruesa es la correspondiente al manto central, seguido de las otras dos zonas que complementan el manto del vellón (manto posterior y manto anterior), así como de la zona del cuello; contrariamente a las zonas del miembro posterior, barriga y del miembro anterior que tienen menor proporción de fibras finas, pero mayor de fibras gruesa. De otro lado, 

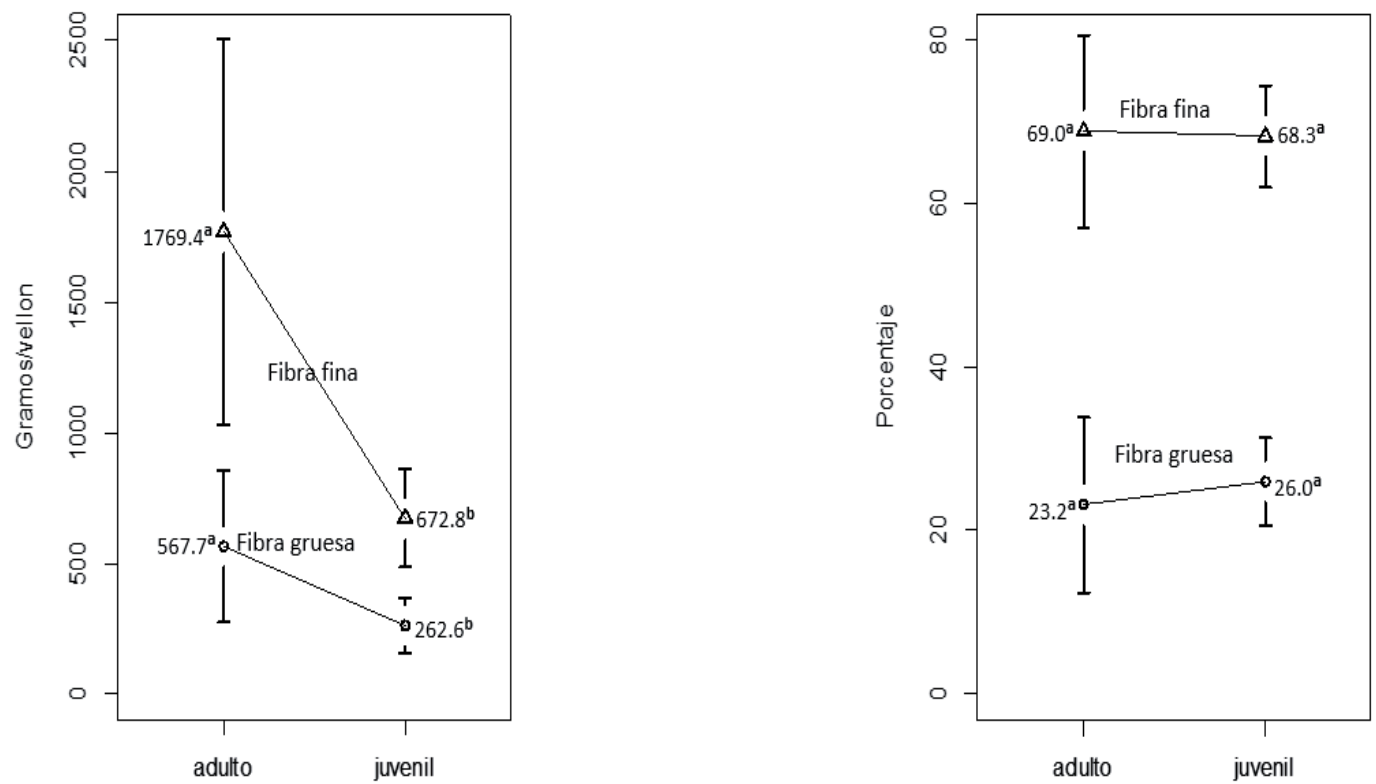

Figura 1. Gráfico de medias e intervlos de confianza del rendimiento al descerdado manual de fibra fina y fibra gruesa en vellones de llama Chaku, por estrato etáreo, expresado en gramos (a la izquierda) y en porcentaje (a la derecha). (A la prueba de Duncan valores con diferentes letras en superíndices tienen diferencia significativa a un valor de $\mathrm{p}<0,05$, dentro de cada tipo de fibra) (Graphic oa average and confidence intervals of the yield to the manual dehairing in down and coarser fiber at Chaku llama fleeces by age. stated in grams (at left) and in percentages (at right). (In each type fiber, values with different superscripts differ significatly to $p<0.05$, at Duncan test)).

Tabla III. Efecto de las regiones corporales sobre el rendimiento al descerdado manual de fibra fina, fibra gruesa e impureza en vellones de llama Chaku (Effect of body sites on the yield to the manual dehairing in down fiber, coarser fiber and impuritie at Chaku llama fleeces).

\begin{tabular}{|c|c|c|c|c|c|c|c|}
\hline \multirow{3}{*}{ Región } & \multirow[t]{2}{*}{$\mathrm{N}$} & \multicolumn{2}{|c|}{$\begin{array}{c}\text { Fibra fina } \\
\text { Promedio } \pm E E\end{array}$} & \multicolumn{2}{|c|}{$\begin{array}{l}\text { Fibra gruesa } \\
\text { Promedio } \pm E E\end{array}$} & \multicolumn{2}{|c|}{$\begin{array}{c}\text { Impureza } \\
\text { Promedio } \pm E E\end{array}$} \\
\hline & & $(\%)$ & (g) & $(\%)$ & (g) & $(\%)$ & (g) \\
\hline & & \multicolumn{2}{|c|}{$* * *$} & \multicolumn{2}{|c|}{$* \star \star$} & \multicolumn{2}{|c|}{ N.S. } \\
\hline Manto central & 15 & $73,45 \pm 1,69^{a}$ & $112,, 5 \pm 11,9^{a}$ & $20,72 \pm 1,56^{c}$ & $30,3 \pm 3,8^{a}$ & $5,83 \pm 0,81$ & $8,5 \pm 1,4$ \\
\hline Manto posterior & 15 & $69,99 \pm 2,44^{\mathrm{ab}}$ & $85,, 8 \pm 16,5^{\mathrm{bcd}}$ & $23,21 \pm 2,35^{b c}$ & $26,1 \pm 4,2^{\mathrm{a}}$ & $6,89 \pm 1,05$ & $8,9 \pm 2,5$ \\
\hline Manto anterior & 15 & $69,71 \pm 2,03^{\mathrm{ab}}$ & $77,, 4 \pm 6,9^{\text {cde }}$ & $23,52 \pm 1,98^{\mathrm{bc}}$ & $27,5 \pm 4,3^{\mathrm{a}}$ & $6,78 \pm 0,92$ & $8,2 \pm 1,6$ \\
\hline Cuello & 15 & $69,36 \pm 2,37^{\mathrm{ab}}$ & $105,, 1 \pm 14,03^{\mathrm{ab}}$ & $23,80 \pm 2,04^{b c}$ & $32,7 \pm 5,3^{a}$ & $6,61 \pm 1,17$ & $10,7 \pm 2,5$ \\
\hline Miembro posterior & 15 & $65,18 \pm 2,53^{\mathrm{bc}}$ & $92,, 7 \pm 17,76^{\mathrm{abc}}$ & $28,46 \pm 2,24^{a}$ & $33,0 \pm 4,5^{a}$ & $6,61 \pm 1,17$ & $9,0 \pm 2,1$ \\
\hline Barriga & 15 & $64,43 \pm 2,26^{c}$ & $54,, 0 \pm 11,22^{\mathrm{e}}$ & $29,02 \pm 2,44^{a}$ & $25,8 \pm 6,2^{a}$ & $6,55 \pm 0,98$ & $6,6 \pm 2,2$ \\
\hline Miembro anterior & 15 & $64,20 \pm 2,34^{c}$ & $64,, 9 \pm 8,97^{\mathrm{de}}$ & $28,46 \pm 2,07^{a b}$ & $27,2 \pm 4,4^{\mathrm{a}}$ & $8,14 \pm 1,42$ & $10,2 \pm 2,4$ \\
\hline
\end{tabular}

*Letras diferentes son estadísticamente significativas a $95 \%$ de confianza con respecto a las columnas.

las zonas del manto, cuello y miembro posterior, debido a que cubren la mayor área del cuerpo producen la mayor cantidad de fibras finas (tabla III), llegando a sumar entre ellas el $80 \%$ de fibra fina.

En cuanto al porcentaje y cantidad de impurezas obtenidas luego del descerdado manual, la zona corporal no tiene efecto, encontrándose impurezas que varían entre el 5,83 y $8,14 \%$.

\section{RENDIMIENTO DE FIBRA FINA EXPRESADO EN PESO/TIEMPO Y EVALUACIÓN ECONÓMICA}

Al descerdado manual, la producción de fibra fina/ persona/h varió desde 5 hasta $21 \mathrm{~g} / \mathrm{h}$, no encontrándose suficiente evidencia del efecto de la región corporal sobre ésta variable (tabla IV), obteniéndose una media global de 9,9 $91,1 \mathrm{~g} / \mathrm{h}$ con una coeficiente de variación alto $(35,81 \%)$, lo que se debería a una alta variabilidad entre personas que trabajaron en el descerdado.
Tabla IV. Rendimiento al descerdado por hora y efecto de las regiones corporales sobre el rendimiento al descerdado manual de fibra fina, cerda e impurezas en vellones de llama Chaku (The yield to manual dehairing per hour and effect of body regions on the yield of manual dehairing in down fiber, coarser fiber and impurities at Chaku llama fleeces).

\begin{tabular}{lrrrrrr}
\hline Región & N & g/ho & DE & $\mathrm{m}$ & $\mathrm{M}$ & $\mathrm{EE}$ \\
\hline Barriga & 10 & $9,6^{\mathrm{a}}$ & 3,9 & 5,3 & 19,0 & 1,2 \\
Cuello & 10 & $10,2^{\mathrm{a}}$ & 4,0 & 5,6 & 19,5 & 1,3 \\
Miembro anterior & 10 & $11,5^{\mathrm{a}}$ & 5,0 & 5,5 & 21,9 & 1,6 \\
Miembro posterior & 10 & $9,1^{\mathrm{a}}$ & 3,4 & 3,7 & 16,9 & 1,1 \\
Manto anterior & 10 & $10,1^{\mathrm{a}}$ & 3,2 & 4,8 & 15,9 & 1,0 \\
Manto central & 10 & $9,7^{\mathrm{a}}$ & 2,5 & 5,7 & 13,9 & 0,8 \\
Manto posterior & 10 & $8,9^{\mathrm{a}}$ & 2,7 & 5,5 & 13,2 & 0,9 \\
\hline
\end{tabular}

*Letras diferentes son estadísticamente significativas a $95 \%$ de confianza con respecto a las columnas. DE= Desviación estandar; m= Mínimo; M= Máximo; EE= Error estandar. 
Tabla V. Análisis económico del proceso de descerdado en vellones de llama Chaku (Economic analisys of manual dehairing process at Chaku llama fleeces).

\begin{tabular}{lc}
\hline Conceptos & Cuantificación \\
\hline Rendimiento de fibra fina/h $(\mathrm{g})$ & 9,9 \\
Rendimiento de fibra fina/día $(\mathrm{g})$ & 78,9 \\
Rendimiento de fibra fina/mes $(\mathrm{kg})$ & 1,9 \\
Remuneración mínima vital (USD \$) & 267,9 \\
Costo de mano de obra/kg de fibra fina (USD \$) & 141,5 \\
Costo de fibra obtenida luego de esquila/kg (USD \$) & 0,9 \\
Otros costos (USD \$) & 3,6 \\
Costo total/kg fibra fina obtenida al descerdado & 145,9 \\
(USD \$) & 7,1 \\
Precio en el mercado/kg fibra fina (USD \$) & $-138,8$ \\
Utilidad neta (USD \$) & 0,05 \\
Relación Beneficio/Costo & \\
\hline
\end{tabular}

Considerando dicho rendimiento, se ha realizado un análisis económico (tabla V), en donde resalta que la producción de $1 \mathrm{~kg}$ de fibra fina al descerdado manual tendría un costo en mano de obra de 141,45 USD \$, y que considerando los costos variables, $1 \mathrm{~kg}$ de fibra fina de llama costaría 145,92 USD \$, lo que contrasta con el precio de fibra fina de llama que se paga en el mercado, el cual apenas llega a los 7,14 USD \$, por lo que se obtiene en vez de utilidad una pérdida de 138.78 USD \$ por kg de fibra descerdada, con una relación Beneficio/ Costo, muy inferior a uno (0,05 unidades).

\section{DISCUSIÓN}

De los resultados obtenidos podemos indicar que las llamas tienen alta proporción de fibra fina, y que lamentablemente ésta no es aprovechada adecuadamente (Stemmer et al., 2005), debido a la falta del proceso del descerdado, del cual también se obtiene fibras gruesas que pueden servir como materia prima. Esto conlleva a indicar que luego del descerdado un uso adecuado de la fibra de llama, sería la utilización de fibras finas para la producción de prendas de vestir de alta calidad, mientras que las fibras gruesas deberían ser derivadas para la confección de alfombras y recuerdos elaborados artesanalmente (tales como llaveros, posavasos, fundas, ganchos, entre otros). De este modo el aprovechamiento de la fibra de llama alcanzaría el $93 \%$.

Nuestro hallazgo resulta similar a lo reportado por Cardozo et al. (2007), pero es menor a la proporción de fibras finas, encontrado por Stemmer et al. (2005), quienes reportan un rendimiento de 91,3\% (aunque no especifican el tipo de vellón), sin embargo dicha diferencia se debería a la metodología aplicada y al tipo de vellón. De otro lado, resulta ser mayor al rendimiento de fibra fina en vicuñas (Adot et al., 2008) y cashemere (McGregor, 2004).

En referencia a la cantidad de fibra/vellón, nuestro resultado es muy similar a lo encontrado por Cochi (1999) y por Stemmer et al. (2005), quienes encontraron una producción de alrededor $1,77 \mathrm{~kg}$, habiendoconsiderando llamas entre 2 y 12 años. De cualquier manera, independientemente de la producción de fibra, el porcentaje de fibra fina obtenida luego del descerdado resulta ser muy similar para todos los vellones, demostrándose en nuestro trabajo que la fibra fina corresponde al $68,60 \pm 2,54 \%$.

De este modo se ha podido constatar que luego del descerdado, aunque existen diferencias del peso de fibra fina, fibra gruesa e impurezas por efecto del estrato etáreo $(p<0,01)$, sin embargo el rendimiento de fibra fina, fibra gruesa e impureza resultan proporcionalmente similares entre animales juveniles y adultos (figura 1), resultado que es similar a lo encontrado por Cochi (1999). De este modo podemos indicar que el crecimiento en longitud y diámetro de fibra fina y la fibra gruesa se genera en una similar proporción, vale decir que aunque las fibras gruesas crecen más longitudinal y transversalmente que las fibras finas, pero como en cantidad es inferior a las fibras finas, la proporción de las fracciones se mantienen (Moore et al., 2015), como consecuencia que el aporte de nutrientes a nivel de los folículos pilosos de dichas fibras también resulta ser proporcional a la cantidad y grosor de las fibras (Galbraith, 2010).

La evidencia encontrada del efecto del estrato etáreo sobre el rendimiento de la producción de fibra fina expresada en peso, resulta contradictorio a lo encontrado por Cochi (1999), quien consideró llamas de dos, tres y cuatro años esquilados anualmente, sin embargo en nuestro trabajo se ha considerado animales de primera esquila (ninguno de los animales habían sido esquilados anteriormente), de modo que ello resulta lógico, pues animales adultos habían tenido mayor periodo de crecimiento de fibra que animales juveniles.

De otro lado la evidencia del efecto de la región corporal sobre el rendimiento porcentual al descerdado de fibra fina, quedaría respaldado en función que las regiones donde se encontraron mayores rendimientos de fibra fina (manto central, posterior y anterior) tienen fibras con menor diámetro, lo cual ya fue demostrado en alpacas por McGregor et al. (2012), en cabras Angora por McGregor (2004), en vicuñas por Quispe et al. (2014) y en llamas por Martínez et al. (1997).

Como la eficiencia del rendimiento al descerdado manual para la obtención de fibra fina de llamas es bajo $(78,9 \pm 9,1 \mathrm{~g} /$ persona / día) respecto el rendimiento de 1300 a $2100 \mathrm{~g} /$ persona/día encontrado por Cochi (1999), cabría indicar que desde el punto de vista económico no sería práctico la implementación de dicho proceso para un aprovechamiento adecuado de dicha fibra, vale decir para un uso de la fibra de llama no solo para sogas, alfombras, recuerdos, entre otros, sino también para la confección de prendas de vestir finas, sin embargo esto podría ser justificable desde un punto de vista social, ya que se presenta como alternativa para la creación de una nueva fuente de trabajo, tan escasa en los lugares donde se realiza la crianza de llamas. Asimismo la obtención de fibra fina y gruesa producto del descerdado puede constituir la oportunidad de tener materia prima para la producción artesanal de prendas de vestir, alfombras y recuerdos, a la que podrían dedicarse principalmente las madres de familia y así mejorar no solo los ingresos familiares, sino también 
mejorar el autoestima de las mismas, al constituirse también en aportantes en la economía familiar.

Ya que la eficiencia al descerdado manual el rendimiento en fibra fina es baja, convendría la implementación del descerdado mecánico en una mini planta, a fin de obtener mayor cantidad de fibra fina en menor tiempo, el cual prestaría servicios a los productores a fin que puedan tener oportunidad de aprovechar los productos obtenidos del proceso para una producción artesanal antes de una producción industrial, de modo que la generación de recursos económico podría estar dirigido principalmente a los productores y artesanales ligados a la producción llamera.

\section{AGRADECIMIENTOS}

A la Escuela Académico Profesional de Zootecnia de la Universidad Nacional de Huancavelica, por habernos facilitados los vellones de llama para el presente trabajo. Asimismo agradecemos al personal del CIPCS - Lachocc de la UNH por habernos apoyado en la esquila de los animales. También al Dr. Bruce McGregor por su apoyo en la traducción del resumen.

\section{BIBLIOGRAFÍA}

Adot, O.G.; de Cossio, A.P. and Maguirre, A. 2008. Industrialization and commercialization of vicuña, guanaco and llama fibres. In: E. Frank, M. Antonini y O. Toro (Eds.). South American camelids research, volume 2. Wageningen Academic Pub. Netherlands. pp. 359-364.

Bustamante, A.V.; Maté, M.L.; Lamas, H.E.; Giovambattista., G.; Zambelli, A. y Vidal-Rioja, L. 2006. Análisis de diversidad genética en tres poblaciones de llamas (Lama glama) del noroeste argentino. Rev Chil Hist Nat, 79: 175-184.

Cardozo, A. 2007. Camélidos. Ed. Centro de Investigación en Forrajes La Violeta. UMSS. Cochabamba. Bolivia. 467 pp.

Coates, W. and Ayerza, R. 2004. Comparison of llama fiber obtained from two production regions of Argentina. J Arid Environ, 58: 513-524.

Cochi, N. 1999. Determinación del rendimiento y calidad de la fibra descerdada de llamas (Lama glama). Tesis para optar el Título Profesional de Ingeniero Agrónomo. Universidad Mayor de San Andrés. La Paz. Bolivia.

Dalton, J. and Franck, R. 2000. Cashmere, camelhair and other hair fibres. In: R. Franck (Ed.). Silk, Mohair, Cahsmere and other luxury fibres, Woodhead Publishing Ltd and CRC Press LLC. Cambridge. pp. 133-174.

Frank, E.N. 2011. Producción de fibra en camélidos sudamericanos. Avances en su procesamiento y mejoramiento genético. Arch Latinoam Prod Anim, 19: 16-19.

Frank, E.N.; Hick M.V. and Adot, O.G. 2011. Descriptive differential attributes of type of fleeces in llama fiber and its textile consequence. Part 2: consequences of the dehairing process. J Text I, 102: 41-49.

Frank, E.N.; HickM.V. and Adot, O.G. 2012. Determination of dehairing, carding, combing and spinning difference from Lama type of fleeces. Int J Appl Sci Tech, 2: 61-70.
Galbraith, H. 2010. Fundamental hair follicle biology and fine fibre production in animals. Animal, 4: 1490-1509.

Hopkins, H.W. 1993. Speciality fibres and markets. In: A.J.F. Russel (Ed.) Alternative animals for fibre production. Commission of the European Communities. Bruselas. pp. 5-10.

Lupton, C.J.; McColl, A. and Stobart, R.H. 2006. Fiber characteristics of the Huacaya Alpaca. Small Ruminant Res, 64: 211-224.

Martínez, Z.; Iñiguez, L. and Rodríguez, T.C. 1997. Influence of effects on quality traits and relationships between traits of the llama fleece. Small Ruminant Res, 24: 203-212.

McGregor, B. and Butler, K.L. 2008. The effects of cashemere attributes on the efficiency of dehairing and dehaired cashmere length. Tex Res J, 78: 486-496.

McGregor, B. 2004. Quality attributes of commercial cashmere. S Afr Society Anim Sci, 34: 137-140.

McGregor, B. 2012. Properties, processing and performance of rare natural animal fibres: A review and interpretation of existing research results. Ed Rural Industries Research and Development Corporation. Publication 11/150. Geelong. Australia. 118 pp.

McGregor, B.; Ramos, H. and Quispe, E.C. 2012. Variation of fibre characteristics among sampling sites for Huacaya alpaca fleeces from the high Andes. Small Ruminant Res, 102: 191-196.

Moore, K.E.; Maloney, S.K. and Blache, D. 2015. High follicle density does not decrase sweat gland density in Huacaya alpacas. $J$ Therm Biol, 47: 1-6.

Nibikora, I. and Wang, J. 2010. Optimum selection of the openning roller and navel for rotor spun silk/Cashmere blended yarn. Fibres Text East Eur, 18: 35-38.

Pinares, R.; Chipa, L.; Paúcar, R. y Quispe, E.C. 2014. Estudio de la diferencia posty pre descerdado de cinco características textiles de la fibra de llama (Lama glama) Ch'aku. Rev Invest Cien Soc Tecnol, 1:69-77.

Quispe, E.C.; Rodríguez, T.C.; Iñíguez, L. y Mueller, J.P. 2009. Producción de fibra de alpaca, llama, vicuña y guanaco en Sudamérica. Anim Genet Resour Inform, 45: 1-14.

Quispe, E.C.; Sánchez, F.; Bartolomé, J. and Alfonso, L. 2014. Variation of commercially important characteristics among sampling sites for vicuña (Vicugna vicugna mensalis) fleeces. J Camel Sci, 7: 1-14.

R. Core Team. 2014. R: A language and environment for statistical computing. R Foundation for Statistical Computing. Vienna. Austria. http://www.R-project.org/ (18/02/2015).

Rodríguez, T.C. 2007. Producción de fibra de camélidos: Calidad de fibra de llama descerdada y clasificada. En: A. Cardozo (Ed.). Camélidos, Centro de Investigación en Forrajes La Violeta. UMSS. Cochabamba. pp. 361-373.

Sacchero, D. y Mueller, J.P. 2005. Determinación de calidad de vellones de doble cobertura tomando al vellón de vicuña (Vicugna vicugna) como ejemplo. Rev Invest Agropec, 34: 143-159.

Siguayro, R. 2009. Comparación de las características físicas de las fibras de la llama Chaku (Lama glama) y la al paca Huacaya (Vicugna pacos) del Centro Experimental Quimsachata del INIA. Puno. Tesis para optar el grado de M. Sc. UNA La Molina. Lima. Perú.

Stemmer, A.; Zarate, M.; Nuernber, J.; Delgado, M.; Wurzinger, M. y Soelkner, J. 2005. La llama de Ayopaya: descripción de un recurso genético autóctono. Arch Zootec, 54: 253-259.

Valbonesi, A.; Cristofanelli, S.; Pierdominici, F.; Gonzales, M. and Antonini, M. 2010. Comparison of fiber and cuticular attributes of alpaca and llama fleeces. Text Res J, 80: 344-353. 
